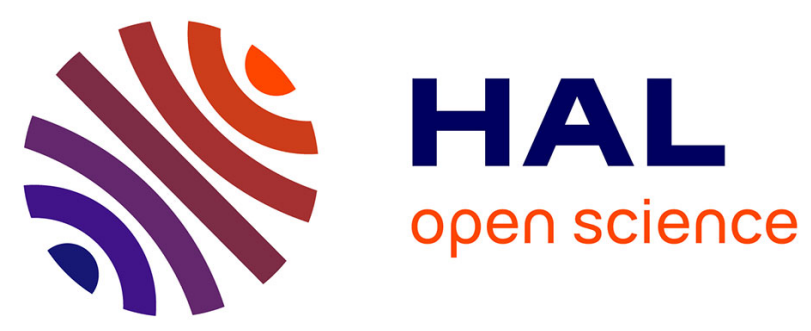

\title{
Application of Ultrasonic Coda Wave Interferometry for Micro-cracks Monitoring in Woven Fabric Composites
}

Pascal Pomarède, Lynda Chehami, Nico Felicien Declercq, Fodil Meraghni, Junliang Dong, Alexandre Locquet, D. S. Citrin

\section{- To cite this version:}

Pascal Pomarède, Lynda Chehami, Nico Felicien Declercq, Fodil Meraghni, Junliang Dong, et al.. Application of Ultrasonic Coda Wave Interferometry for Micro-cracks Monitoring in Woven Fabric Composites. Journal of Nondestructive Evaluation, 2019, 38 (1), pp.26-34. 10.1007/s10921-019-0563z . hal-02317872

\section{HAL Id: hal-02317872 \\ https://hal.science/hal-02317872}

Submitted on 16 Oct 2019

HAL is a multi-disciplinary open access archive for the deposit and dissemination of scientific research documents, whether they are published or not. The documents may come from teaching and research institutions in France or abroad, or from public or private research centers.
L'archive ouverte pluridisciplinaire HAL, est destinée au dépôt et à la diffusion de documents scientifiques de niveau recherche, publiés ou non, émanant des établissements d'enseignement et de recherche français ou étrangers, des laboratoires publics ou privés. 


\title{
Application of Ultrasonic Coda Wave Interferometry for Micro-cracks Monitoring in Woven Fabric Composites
}

\author{
Pascal Pomarède ${ }^{1,2} \cdot$ Lynda Chehami $^{2,3} \cdot$ Nico F. Declercq ${ }^{2,3} \cdot$ Fodil Meraghni ${ }^{1,2} \cdot$ Junliang Dong ${ }^{2,4}$. \\ Alexandre Locquet ${ }^{2,4}$. D. S. Citrin ${ }^{2,4}$
}

\begin{abstract}
The consequences of a four-point bending test, up to $12 \mathrm{~mm}$, are examined by emitting $1 \mathrm{MHz}$ ultrasonic guided waves in woven carbon fiber reinforced polymer specimens, using coda wave interferometry (CWI), revealing a potential use for nondestructive evaluation. It is known that CWI is more sensitive to realistic damage than the conventional method based on the first arriving time of flight in geophysical, or in civil engineering applications such as concrete structures. However, in composite materials CWI is not well established because of the involved structural complexity. In this paper, CWI is investigated for monitoring the occurrence of realistic defects such as micro-cracks in a woven carbon fiber composite plate. The micro-cracks are generated by a four-point bending test. The damage state is stepwise enhanced by gradually increasing the load level, until failure initiation. The damage is monitored, after each loading, using ultrasound. It is demonstrated that CWI is a powerful tool to detect damage, even low levels, in the sample. Two damage indicators based on CWI, i.e. signals correlation coefficient and relative velocity change, are investigated and appear to be complimentary. Under significant loading levels, the normalized cross-correlation coefficient between the waveforms recorded in the damaged and in the healthy sample (reference at $0 \mathrm{~mm}$ ), decreases sharply; this first indicator is therefore useful for severe damage detection. It is also demonstrated, by means of a second indicator, that the relative velocity change between a baseline signal taken at zero loading, and the signals taken at various loadings, is linear as a function of the loading, until a critical level is reached; therefore this second indicator, is useful for low damage level detection. The obtained evolution of the relative velocity measurement is supported by relative comparison to the evolution of the bending modulus in function of displacement. The relative velocity change exhibits the same evolution as the bending modulus with loading. It could be used to indicate when the material stiffness has decreased significantly. The research is done in the framework of composite manufacturing quality control and appears to be a promising inspection technique.
\end{abstract}

Keywords Coda wave $\cdot$ Non-destructive evaluation $\cdot$ Composite materials $\cdot$ Ultrasonic waves

\section{Introduction}

Thanks to their advantages of light weight, design flexibility, and high specific stiffness and strength, composite materials have been widely used for several years as structural materials in a number of industrial sectors ranging from aviation, automotive [1,2], civil construction, and the sport industry [3-5]. Nondestructive testing and inspection of composite structures, both for manufacturing quality assurance and for in-service damage detection, has, over the last years, prompted the development of a number of techniques including water-and air-coupled ultrasound, bond testing, thermography, shearography and terahertz imaging [6]. A review of the mentioned methods is given by Gholizadeh 
[7]. Those methods were used either to detect the location of the appearing damage or to quantify its influence on the mechanical properties of the tested specimens $[8,9]$.

The field of nondestructive evaluation (NDE) concentrates on the detection of significant defects, as well as material damage in early stages of degradation. Generally, the detection of the defect is determined by looking at the impulse response of the medium which can be used to image a complex medium. In standard techniques, the complete waveform is obtained with an active configuration in which an ultrasonic wave is generated at one location on the specimen and recorded at another position. In more complex media where multiple scattering and sound diffusion may occur, a novel monitoring technique has been developed in geoscience to measure the relative velocity change of different seismic waves, called coda wave interferometry (CWI) [10].

After propagation of elastic waves in the medium, they interact several times with the several minor defects. The late part of the diffuse field is termed the "coda". The term coda originates from musical science [11] and was adopted in seismology later to refer to the CWI technique. CWI was named by Snieder [10] where they proved the possibility of detecting weak velocity changes in solids using seismic and ultrasonic coda waves. In this CWI technique, the main focus is thus given to the late part of the recorded waveform, the coda. CWI has since then successfully been used in seismology and acoustics $[12,13]$. An ultrasonic wave introduced into a complex medium experiences multiple scatterings and becomes a diffuse field. A transmitted sound field can be separated into two parts: the first arrival, which consists of waves that directly travel along the path from the source to the receiver or basic reflection path than can be easily distinguished, and the diffuse part which includes the late coda contribution. The diffuse waves travel much longer through the medium than the first arrival, due to multiple scattering $[14,15]$. The later the arrival, the longer the path travelled and the tighter and completer the occurred interaction with the micro-structure. Because of the longer paths, diffuse waves are more sensitive to minor changes in velocity and constitutive properties in the medium. Consequently, diffuse waves carry more micro-structural information than the first wavepackets and it is crucial to find a technique to extract that information. The CWI technique or seismic doublet method $[16,17]$ compares two different time series of coda elastic waves and determines their degree of correlation. The technique is widely used in geophysics and civil engineering [18]; however, its application for composite materials inspection is relatively new.

Coda waves in composite specimens originate naturally through multiple scattering by heterogeneities and fibers. Hence, one may expect the coda to be more sensitive to encountered material damage. Recently, Zhu et al. [17] have used coda waves to determine internal stress in a polymer composite. Livings et al. [19] investigated the feasibility of coda waves to estimate the thermally induced ultrasonic velocity variation in a plexiglas plate and in a carbon fiber reinforced polymer composite plate.

In the literature, most studies concern fatigue under cyclic tensile or bending loading and have shown that, under successively increasing stress, matrix cracking is the first type of damage appearing in composites [20,21]. These cracks, forming in the host medium (resin or matrix), gradually reach areas between the fibers. In the specific case of cyclic continuous loading, the cracks grow in the matrix and fiber/matrix interface until they reach the fibers of an adjacent layer. If the stress concentrations are sufficiently large, the fibers break; otherwise, the cracks propagate along the fiber/matrix interface of the adjacent ply causing delaminations [22]. In this report, we investigate the capability of a nondestructive method based on CWI to quantify micro-crack growth in a woven carbon fiber reinforced polymer composite. The paper is organized as follows. In the next section, the CWI theory is presented. The experimental procedure to introduce damage into a composite sample via a four-points bending test is then detailed. The evolution of the bending modulus is also calculated for a first estimation of the induced damage. The ultrasonic acquisition setup and the data processing method are then presented. The evolution of the relative velocity of the coda waves when increasing the bending loading level is computed and compared to the aforementioned bending modulus decrease. A discussion of the advantages of the method is presented at the end.

\section{Detection of Four-Point Bend Induced Damage via Coda Wave NDE}

\subsection{Coda Wave Interferometry Theory}

Consider two time-invariant signals $x_{1}(\mathrm{t})$ and $x_{2}(\mathrm{t})$, where $x_{1}(\mathrm{t})$ designates the reference signal (without loading) and $x_{2}(\mathrm{t})$ the signal recorded in the damaged case (after propagation in the loaded medium). The changes in the waveforms can be quantified by computing the normalized time shifted cross-correlation parameter $R\left(t_{s}\right)$ over a short-time window of length $T$ centered at time $t^{\prime}$ [17]:

$$
R\left(t^{\prime}, t_{s}\right)=\frac{\int_{t^{\prime}-T / 2}^{t^{\prime}+T / 2} x_{1}\left(t+t_{S}\right) x_{2}(t) d t}{\sqrt{\int_{t^{\prime}-T / 2}^{t^{\prime}+T / 2} x_{1}^{2}\left(t+t_{S}\right) d t \int_{t^{\prime}-T / 2}^{t^{\prime}+T / 2} x_{2}^{2}(t) d t}},
$$

where $t_{s}$ is the time shift between the perturbed and unperturbed waveforms, respectively. This normalized cross- 
correlation, or temporal coherence as called by Michaels and Michaels [23], provides an amplitude independent measure of the similarity in the shape of the two signals when the second signal is delayed by $t_{s}$.

If the signal $x_{2}(t)$ is not perturbed, $x_{1}(t)$ and $x_{2}(t)$ have identical shape within the window centered at $t^{\prime}$,

$x_{2}(t)=x_{1}(t)$

and $R\left(t^{\prime}, t_{s}=0\right)=1$.

When $x_{2}(t)$ is a $\delta t$ time shifted version of the original wave, then

$x_{2}(t)=x_{1}\left(t+t_{s}\right)$,

and $R\left(t^{\prime}, t_{s}\right)$ reaches its maximum for $t_{s}=\delta t$.

Usually, the time lag $\delta t$ is assumed to evolve linearly with time when the effective velocity change is homogeneous over the whole material, i.e.,

$\delta t=-\frac{\delta V}{V} t$

When the effective velocity change is local, or at least not distributed over the area where the CWI method is sensitive, the time lag could deviate from a linear evolution. This relation is experimentally verified in several works when considering a pure dilatation of the materials under thermal effects [19,24] or studying weak stress effect in heterogeneous samples such as concrete [25].

\subsection{Experimental Setup}

The material used in this study is a polypropylene sulfide based composite, reinforced with eight layers of woven carbon fibers with the following layering : $\left[0^{0} / 90^{0}\right.$, $\left.-45^{0} / 45^{0}, 0^{0} / 90^{0},-45^{0} / 45^{0}\right]_{s}$. Two specimens of dimensions $160 \mathrm{~mm} \times 25 \mathrm{~mm} \times 2.4 \mathrm{~mm}$ along the warp and weft direction $\left(0^{0} / 90^{0}\right)$ are cut from one plate of $300 \mathrm{~mm} \times$ $300 \mathrm{~mm} \times 2.4 \mathrm{~mm}$, using a water jet cutter. Young's modulus is $56 \mathrm{GPa}$ along the fiber's axes in both warp and weft directions. The first specimen was subjected to a four-point bending test under static loading before each ultrasonic measurement. The second one however, is used as a reference to monitor possible changes in the coda wave signals induced by variations in environmental condition.

\subsubsection{Four-Bend Loading Tests}

The applied displacement load is increased from 2 to $12 \mathrm{~mm}$ in 11 steps of $1 \mathrm{~mm}$ with ultrasonic acquisition after each displacement increment. The applied force values are indicated in Table 1.

The sample is loaded at a constant displacement rate of $24 \mathrm{~mm} \mathrm{~min}^{-1}$ at room temperature, on a tensile test machine (Zwick Roell Z050) and in compliance with the ISO 5893 standard. The samples exhibit a response that remains near linear as shown in Fig. 1.

This introduces a damage increase with increasing displacement. A representation of the four points bending set-up is shown in Fig. 2.

In order to have a first estimation of the induced damage, the bending modulus reduction with applied loading displacement has been calculated. The damage is calculated as $D=1-E_{n} / E_{0}$, with $E_{n}$ the unloaded bending modulus for each considered applied displacement and $E_{0}$ the bending loading modulus measured for the first bending test. The obtained damage evolution function of the applied displacement is plotted in Fig. 3. Three main steps may be distinguished. First, from 0 to $3 \mathrm{~mm}$ of applied displacement, no significant evolution of the bending modulus is noted yet. During this step the cracks initiate in the specimen. Then a close to linear evolution of the damage up to 0.13 is noticeable until $8 \mathrm{~mm}$. The evolution of damage then stabilizes at 0.13 for an applied displacement from 9 to $11 \mathrm{~mm}$. This is induced by a saturation of progressive damage. Finally, the applied displacement of $12 \mathrm{~mm}$ corresponds to the occur-

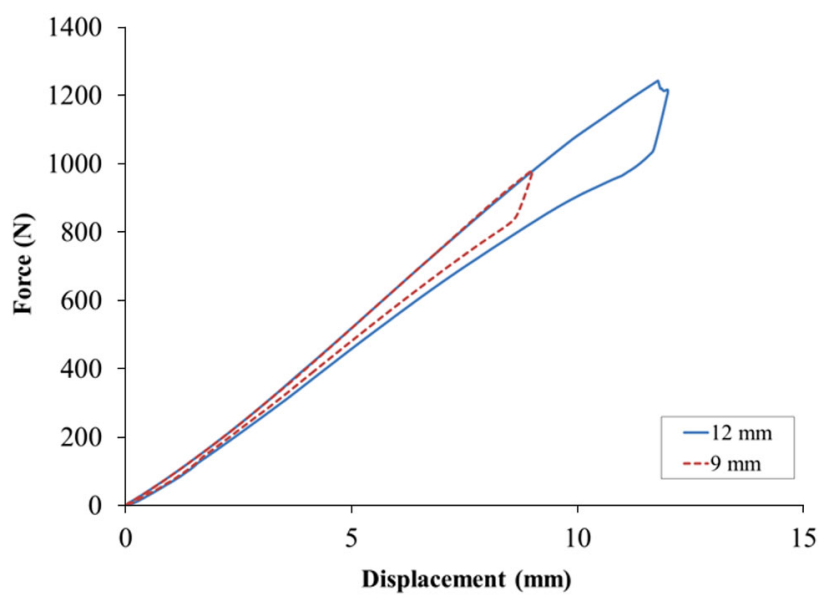

Fig. 1 Force-displacement diagram. Example of $9 \mathrm{~mm}$ (dashed line) and $12 \mathrm{~mm}$ (continuous line)
Table 1 The values of displacements and loads for static loading

\begin{tabular}{llllllllllll}
\hline Displacement $(\mathrm{mm})$ & 2 & 3 & 4 & 5 & 6 & 7 & 8 & 9 & 10 & 11 & 12 \\
\hline Force $(\mathrm{N})$ & 187 & 292 & 399 & 519 & 638 & 761 & 876 & 978 & 1100 & 1200 & 1240 \\
\hline
\end{tabular}


(a)

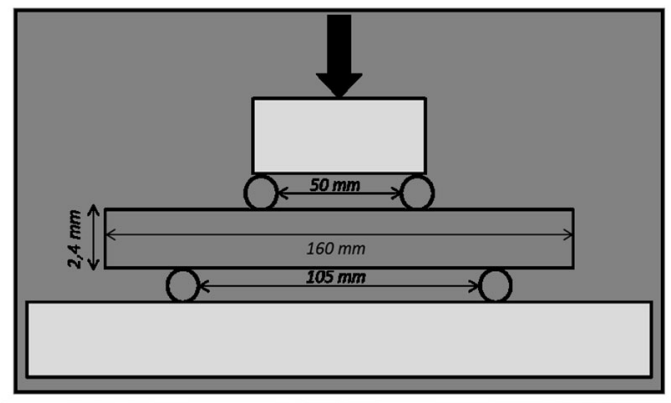

(b)

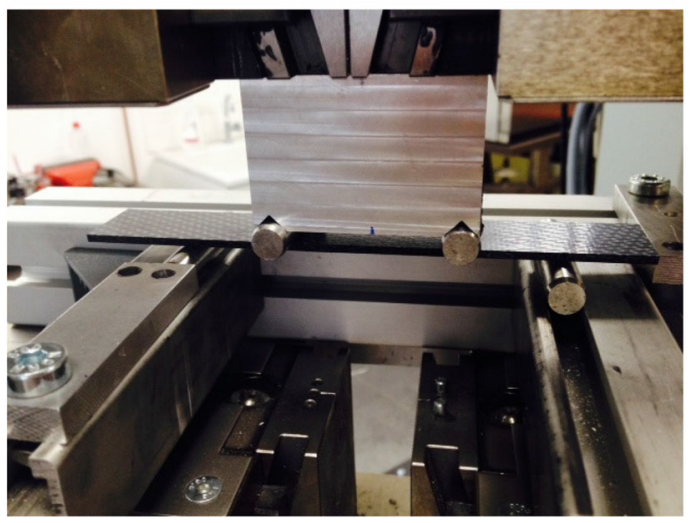

Fig. 2 a Schematic view of the four-bend configuration used to generate micro-cracks. b Photograph of the four-bend configuration

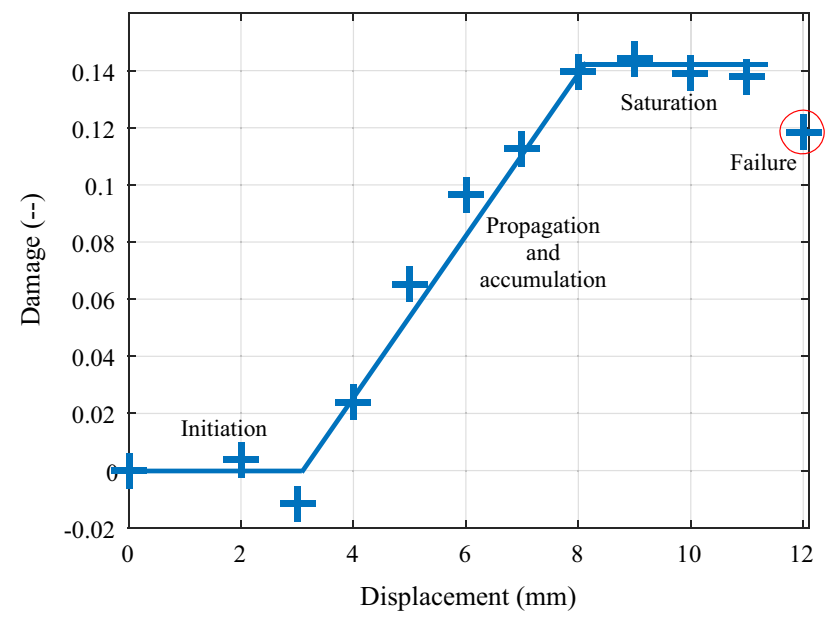

Fig. 3 Evolution of damage, calculated as the reduction of the bending modulus $\left(D=1-E_{n} / E_{0}\right)$, while increasing the magnitude of the applied four points bending displacement

rence of large cracks in the sample surface, consequently the value of induced damage for this displacement should be considered with caution.

\subsubsection{Ultrasonic Measurements}

Two ultrasonic measurements are performed after each test, namely for the sample subjected to bending test and for the reference sample, respectively. Two Panametrics sensors, polarized longitudinally, having $1 \mathrm{MHz}$ PZT disks, and of

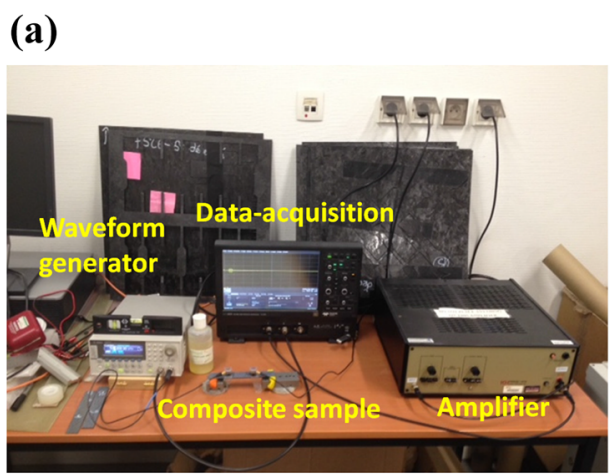

(b)

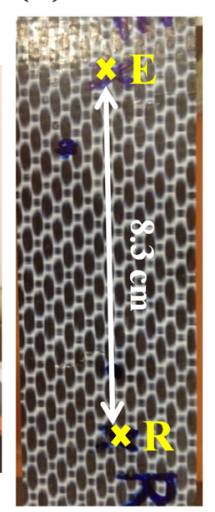

Fig. 4 a Photograph of the ultrasonic experimental configuration. b Zoom on the sample

$12.5 \mathrm{~mm}$ wide, are used. The first transducer is powered by a waveform generator (Agilent Technology model) and is excited by a sine burst of 1 cycle at a frequency of $1 \mathrm{MHz}$ and amplitude of $10 \mathrm{~V}$ peak-to-peak. The generated elastic waves are recorded by a second transducer of the same frequency. The ultrasonic experimental setup is shown in Fig. 4.

The total length of the signals is $1000 \mu \mathrm{s}$, which is sufficient to capture most of the energy in the ultrasonic field. Indeed, as it can observed in Fig. 5, the attenuation in the composites is high, specifically for this given frequency, when we get closer to $1000 \mu \mathrm{s}$ and this may lead to inaccurate results from cross-correlation. Both transducers are coupled (universal Sofranel coupling Gel-D) on the top surface of the plate and spaced by $83 \mathrm{~mm}$. The received signals are amplified by $100 \mathrm{~dB}$ and averaged over 250 measurements before being recorded by an oscilloscope.

\subsection{Data Processing}

As mentioned in Sect. 2.2 for the sample subjected to bending, the applied displacement load is increased from 2 to $12 \mathrm{~mm}$ in 11 steps of $1 \mathrm{~mm}$ with ultrasonic acquisition at each displacement increment. In Fig. 5 two typical signals obtained for $0 \mathrm{~mm}$ and $3 \mathrm{~mm}$ are shown. Both signals present an rapid decrease from $150 \mu \mathrm{s}$, showing a good ultrasonic coda appearance. For the first arrival signal, i.e. in the range of $\left[\begin{array}{ll}0 & 150\end{array} \mu \mathrm{s}\right.$, the waveforms are relatively similar in both phase and amplitude as it was verified by means of cross-correlation on first arrival signal. The small difference in amplitude and phase of the first arrival can be explained by the fact that the coupling of the transducers changes slightly from one measurement to the next. However, for the later interval (coda) the signals are distinct, where one of the waveform is a time shifted version of the other.

Before defining the size of the coda windows that will be investigated further, all the recorded signals of $1000 \mu$ s long 


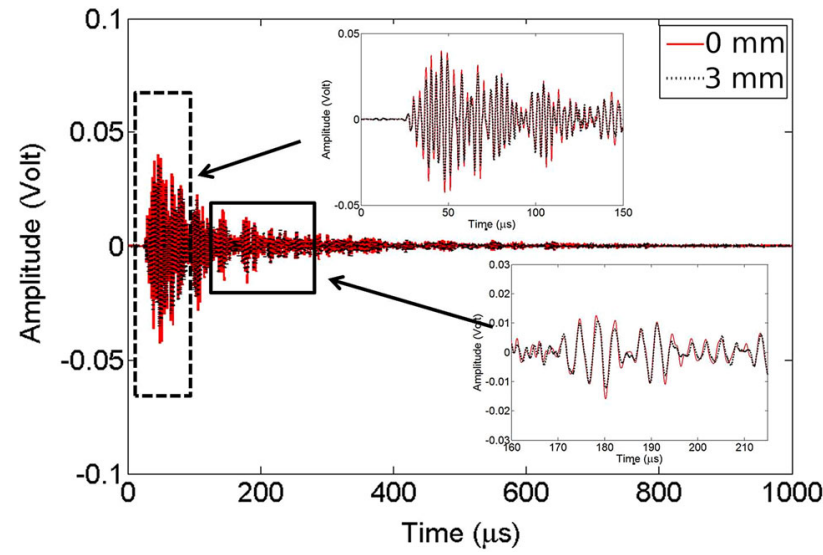

Fig. 5 Variation of the amplitude versus time of the reference waveform recorded before applying load (continuous line) compared with that recorded after a change in load of $3 \mathrm{~mm}$ (dashed line). The first arrivals $[0,150] \mu \mathrm{s}$ are relatively similar in both amplitude and phase. A time shift is observed however between the coda parts $[160,215] \mu$ s of the reference and the damaged cases

were all adjusted in time in order to have the first wave packet arrival at the same time than for the reference signal. When repeating ultrasonic measurements of samples undergoing physical changes, for instance by loading, it is important to note that there will be a slight difference between positioning two transducers at an exact Euclidean distance from one another, and positioning two transducers each at their original spot on the sample. The reason is that if the sample is deformed, the original spots on the sample may change in Euclidean distance from each other. Just as with the use of the speed of light as absolute reference, we have opted for an off-set correction based on the first arrival of sound in the experiments. Therefore, small differences caused by either imperfect positioning of the transducers on the sample or by changing the distance between those positions due to sample deformation caused by the applied bending tests, are automatically corrected. Making an off-set correction is allowed because the coda is characterized not by its actual time delay but by a time dependent time delay.

Now, because the procedure is based on a calculation of correlation between the reference and the measured signal in limited sections of the entire coda wave time windows, section by section, it is important to ensure that each shifted part of the coda signal stays as much as possible within the considered section during the procedure and therefore we must minimize the off-set. Indeed, this is precisely what is done here, namely by correcting the off-set, based on the first arrival of the received signal. Indeed, the two transducers were positioned by hand after each four points bending tests on the sample on marks draw in the samples. The offset correction was done to reduce any possible influence of imperfect transducer positioning, or slight sample size alteration due to the performed loading, that could interfere with a correct estimation of relative velocity change. Note however that, despite our care in this respect, a small off-set correction hardly visible to the naked eye was applied to some of the raw signals. There was indeed never any major shift of the first wave arrivals after applied bending compared to the reference signal.

As shown in Fig. 5, the coda part appears after the first arrivals $(t>150 \mu \mathrm{s})$. In this paper, we consider the coda as the part of the signal in the interval $[200,600] \mu$ s where the attenuation is still limited. It is a compromise between sufficiently multi-scattering waves (beginning of the window must therefore be sufficiently late in the signal) and the signal-to-noise ratio (end of the window should not be too much attenuated). The choice of the coda part is important to avoid apparent large fluctuations early and late in the assumed coda window.

Here, all the raw signals (coda part) have the same total length $T_{t}=400 \mu \mathrm{s}$ and sampling time $\Delta t=0.002 \mu \mathrm{s}$. As mentioned in $[16,19]$ when the perturbation in the sample is small, the relative velocity change of the coda part in the signal can be considered as constant. In this configuration the time lag increases linearly with the arrival time. As a first hypothesis the damage introduced by four points bending will be considered to match this hypothesis. This will be verified with the first experimental results. As the relative velocity change is constant between two time signals (reference and damaged case for corresponding loading value), the time lag will increase linearly with the arrival time. Hence, for accurate estimation of $\delta t$, we must consider a short time correlation window section of length $T$ in such a way that $\delta t$ is as constant as can be inside this window section. Commonly, $T$ must satisfy the coda interferometry hypothesis $T * \Delta f \gg 1$, where $\Delta f$ is the frequency band of the coda signal [26]. In our case, the frequency band of the coda signals is about $400 \mathrm{kHz}$, so for a $\mathrm{T}$ equal to $T * \Delta f=1$ we have to choose $T$ at least $2.5 \mu \mathrm{s}$. Here, $T$ is set to $50 \mu \mathrm{s}$. In order to satisfy the interferometry hypothesis, the normalized cross-correlation coefficient $R\left(t^{\prime}, t_{s}\right)$ is then computed by using Eq. 1 . Hence, $R\left(t_{s}\right)$ is computed for each window by shifting this time window over each $t_{s}$ increment $(\Delta t)$. Note that the reference signal here is the waveform recorded without loading $(0 \mathrm{~mm})$. An example of $R\left(t^{\prime}, t_{s}\right)$ is shown in Fig. 6.

For each ultrasonic measurement, $R$ is computed by moving the window by $T / 2$ in the coda signals. The normalized cross-correlation coefficients and $\delta t$ versus the time obtained for both samples are shown in Fig. 7.

In Fig.7a, from 2 to $11 \mathrm{~mm}$ all cross correlation coefficients exhibit the same evolution with time window. In addition, the coefficient value is always around 0.8 , which means that the signals have a high similarity with the reference signal $(0 \mathrm{~mm})$. An important decrease of the coefficient is, however, noticed for the signal recorded at $12 \mathrm{~mm}$. In 

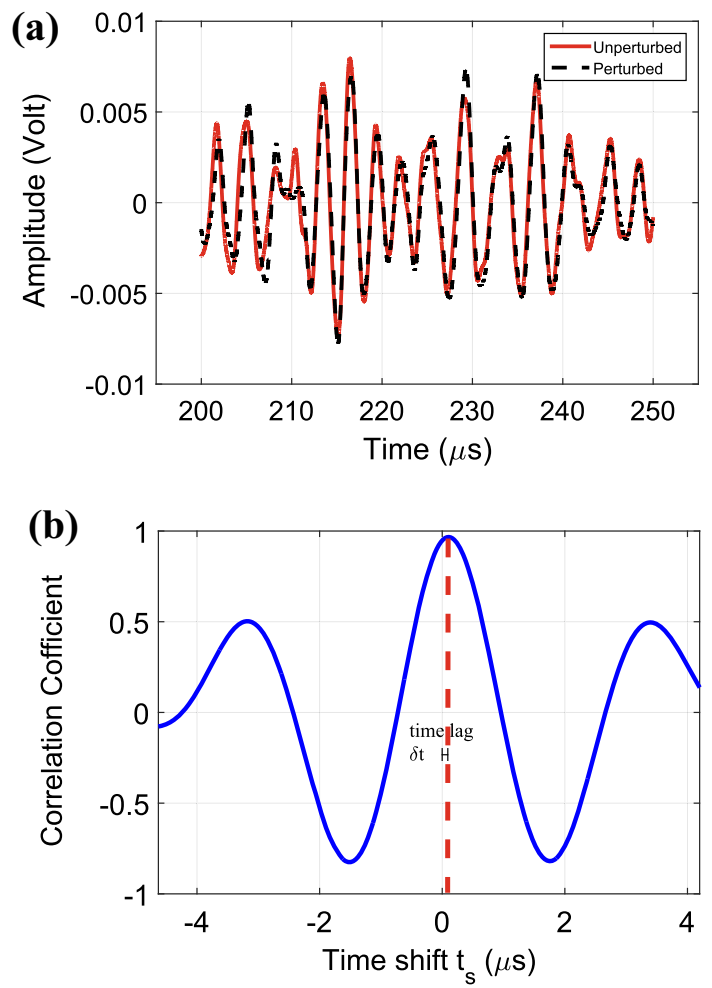

Fig. 6 a Example of time window signals obtained for the reference (continuous line) and perturbed signal obtained for $3 \mathrm{~mm}$ (dashed line). b Time lag $\delta t$ estimation using the cross-correlation coefficient between both windowed signals

addition, its evolution with time window is very different from the other signals. This can be explained by the appearance of large cracks on the surface of the sample. Therefore, no time lags were extracted from this signal. Similar trends can be observed in Fig. $7 \mathrm{~b}$ for the reference sample where it decreases until 0.6. Under these observations, a small variation of the environmental conditions (temperature for example) may induce significant fluctuations on the crosscorrelation coefficient. Thus, the useful information from small defects can not be extracted directly from the crosscorrelation coefficient corresponding to the damaged sample.

In Fig. 7c, the time lag $\delta t$ of all signals obtained for different loading values exhibit an evolution near linear with the time window which match the hypothesis made earlier about the appearance of small perturbation in the specimen, at least until $11 \mathrm{~mm}$ of applied displacement. The associated linear coefficient also increases with the applied loading level. This was confirmed visually in Fig. $7 \mathrm{c}$ and by linear curve fitting. However, in Fig. 7d, the time lag does not show significant changes. Consequently, the time lag variation is more sensitive to the occurrence of micro-cracks inside the material. Hence, this parameter can be used complementary to distinguish the signature of the defect from the environmental conditions.
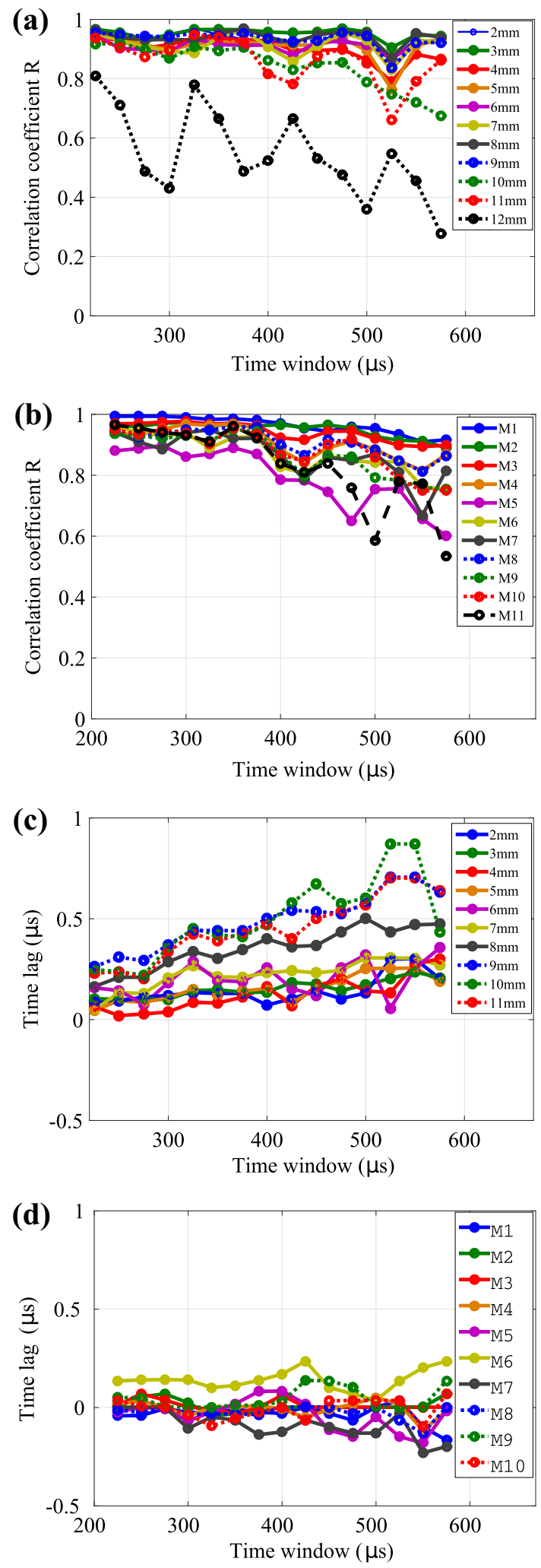

Fig. 7 a Normalized cross-correlation coefficient $R$ between the waveforms recorded in the damaged case $(2-12 \mathrm{~mm})$ and the reference $(0 \mathrm{~mm})$. b $R$ for the sample of reference (environmental conditions) for different measurements M. $\mathbf{c}$ and $\mathbf{d}$ Corresponding time lag $\delta t$ for the damaged sample and the reference sample, respectively versus time window 


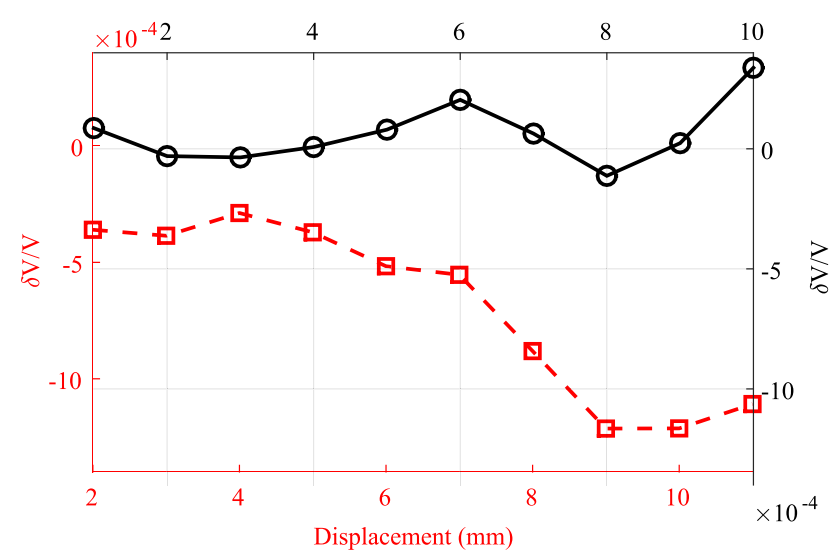

Fig. 8 Relative velocity changes obtained for different loading values (dashed line) and the reference sample (continuous line). The measurements on the reference sample (without induced damage) were made at the same time as for the sample submitted to loading

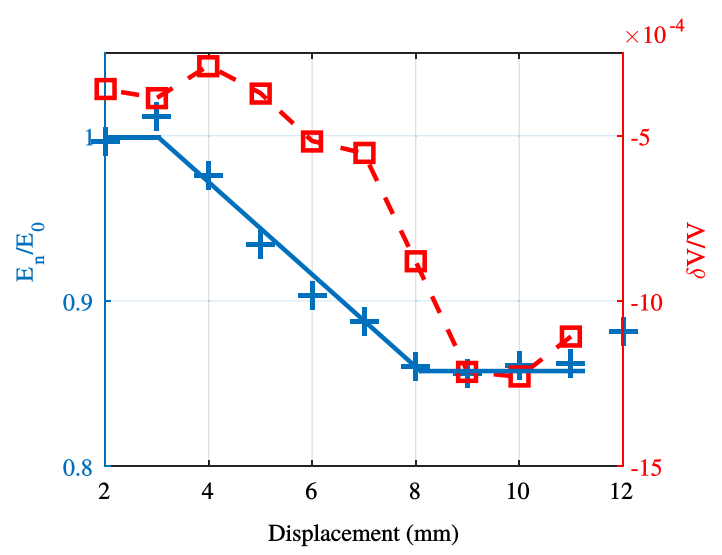

Fig. 9 Relative velocity changes (dashed line) and the normalized bending modulus evolution (continuous line) obtained for different loading values

To estimate the velocity change, $\delta t$ is then used. In this paper, the slope $\delta t / t$ is estimated by taking an average of $\delta t / t$ in the interval $[200,600] \mu s$. This processing is similar, for example, to what described is by Zhu et al. [17] for a glass/epoxy composite sample under compression tests. The entire procedure is repeated for every sample (under loading and environmental conditions). The relative velocity variation $\delta V / V=-\delta t / t$ was computed and is shown in Fig. 8 .

Figure 8 shows plots of the velocity changes versus loading values for the two woven carbon fiber composite plates. Both the results from the sample subjected to bending and the sample kept undamaged are indicated. For the second sample, kept intact (continuous line in Fig. 8), relatively low evolution of the relative velocity is noticed. The value change oscillates at low amplitude around 0, except for measurements taken after an applied bending of 7 and of $11 \mathrm{~mm}$ on the other sample. For this sample subjected to bending (dashed line in Fig. 8), the general behavior is that the velocity change decreases near linearly as the damage level increases and beyond an applied displacement of $4 \mathrm{~mm}$ reaches $0.125 \%$ at $9 \mathrm{~mm}$. However after $9 \mathrm{~mm}$, the $\delta V / V$ does not vary anymore and remains around $0.125 \%$ until an applied displacement of $11 \mathrm{~mm}$. The evolution of the $\delta V / V$ is very similar to the decrease of the bending modulus which can be recover from the evolution of damage that is plotted in Fig. 3. In order to confirm it, both the normalized decrease of the bending modulus $\left(E_{n} / E_{0}\right)$ and the relative velocity change are plotted on the same graph in Fig. 9 for comparison purpose. The three steps of the damage indicator evolution can clearly be observed on both cases. They are, first an absence of variation, then a near linear decrease and finally a saturation of the evolution of the damage indicator.

\section{Conclusion}

CWI of diffuse ultrasonic signals has been investigated as a means of detecting and discriminating micro-cracks caused by static loading, inside a woven carbon fiber reinforced composite sample. The correlation coefficient between a waveform measured in the damaged state and a baseline signal is a quantitative measure of how much the similarity (i.e., the shape) has changed compared to the reference signal (healthy state) as a function of time from the transmitted pulse. The evolution of this coefficient shows significant decrease for high values of applied loadings. A quantitative study of the relative velocity change is therefore proposed. The time lag change (or relative velocity change) between a waveform measured in the damaged state and a baseline signal is a quantitative measure of how much the signal is shifted compared to the reference signal (healthy state) as a function of time from the transmitted pulse; this analysis parameter provides the best discrimination for the data shown here. We noticed the beginning of the evolution at $4 \mathrm{~mm}$, then a near linear evolution up to $9 \mathrm{~mm}$, and finally saturation of the damage increase until $11 \mathrm{~mm}$. It was observed that this description of the damage is very similar to the measured decrease of the bending modulus. The bending modulus could be monitored and an alarm could be activated when the decrease reach a limited point when large cracks may have been reaches. The overall methodology is very promising for structural health monitoring because of its sensitivity to small defects, nondestructive aspects and the continuous time monitoring of the health condition of composites. Future investigations should consider samples submitted to fatigue damage; such tests are indeed closer to the solicitation that a structure may undergo in the industrial field.

Acknowledgements The authors acknowledge Vincent Tournat for his scientific discussions. This work is supported by the Région Grand Est. 


\section{References}

1. Malpot, A., Touchard, F., Bergamo, S.: Effect of relative humidity on mechanical properties of a woven thermoplastic composite for automotive application. Polym. Test. 48, 160-168 (2015)

2. Pomarède, P., Meraghni, F., Peltier, L., Delalande, S., Declercq, N.F.: Damage evaluation in woven glass reinforced polyamide 6.6/6 composites using ultrasound phase-shift analysis and X-ray tomography. J. Nondestruct. Eval. (2018). https://doi.org/10.1007/ s10921-018-0467-3

3. Eckel, S., Meraghni, F., Pomarède, P., Declercq, N.F.: Investigation of damage in composites using nondestructive nonlinear acoustic spectroscopy. Exp. Mech. 57(2), 207-217 (2016)

4. Yaacoubi, S., Chehami, L., Aouini, M., Declercq, N.F.: Ultrasonic guided waves for reinforced plastics safety. Reinf. Plast. 61, 87-91 (2017)

5. Cole, W.F., Armstrong, K.B., Bevan, L.G.: Care and Repair of Advanced Composites. SAE International, Warrendale (2005)

6. Dong, J., Kim, B., Locquet, A., McKeon, P., Declercq, N., Citrin, D.S.: Nondestructive evaluation of forced delamination in glass fiber-reinforced composites by terahertz and ultrasonic waves. Composites B 79, 667-675 (2015)

7. Gholizadeh, S.: A review of non-destructive testing methods of composite materials. Procedia Struct. Integr. 1, 50-57 (2016)

8. Marguères, P., Meraghni, F., Benzeggagh, M.L.: Comparison of stiffness measurements and damage investigation techniques for a fatigued and post-impact fatigued GFRP composite obtained by RTM process. Composites A 31(2), 151-163 (2000)

9. Marguères, P., Meraghni, F.: Damage induced anisotropy and stiffness reduction evaluation in composite materials using ultrasonic wave transmission. Composites A 45, 134-144 (2013)

10. Snieder, R.: The theory of coda wave interferometry. Pure Appl. Geophys. 163(2-3), 455-473 (2006)

11. Benward, B., Saker, M.: Music in Theory and Practice, vol. II. McGraw-Hill Education, New York (2009)

12. Poupinet, G., Ellsworth, W.L., Frechet, J.: Monitoring velocity variations in the crust using earthquake doublets: an application to the Calveras Fault, California. J. Geophys. Res. 89(B7), 5719-5731 (1984)

13. Frjd, P., Ulriksen, P.: Amplitude and phase measurements of continuous diffuse fields for structural health monitoring of concrete structures. NDT \& E Int. 77, 35-41 (2016)

14. Becker, J., Jacobs, L.J., Qu, J.: Characterization of cement-based materials using diffuse ultrasound. J. Eng. Mech. 129(12), 14781484 (2003)

15. Aki, K., Chouet, B.: Origin of coda waves: source, attenuation, and scattering effects. J. Geophys. Res. 80(23), 3322-3342 (1975)
16. Sthler, S.C., Sens-Schnfelder, C., Niederleithinger, E.: Monitoring stress changes in a concrete bridge with coda wave interferometry. J. Acoustical Soc. Am. 129(4), 1945-1952 (2011)

17. Zhu, Q., Binetruy, C., Burtin, C.: Internal stress determination in a polymer composite by coda wave interferometry. IOP Conf. Ser. Mater. Sci. Eng. 137, 012040 (2016)

18. Zhang, Y., Abraham, O., Tournat, V., Le Duff, A., Lascoup, B., Loukili, A., Grondin, F., Durand, O.: Validation of a thermal bias control technique for coda wave interferometry (CWI). Ultrasonics 53(3), 658-664 (2012)

19. Livings, R., Dayal, V., Barnard, D.: Coda wave interferometry for the measurement of thermally induced ultrasonic velocity variations in CFRP laminates. AIP Conf. Proc. (2016). https://doi.org/ 10.1063/1.4940588

20. Salkind, M.: Fatigue of composites. In: Composites Materials: Testing and Design (Second Conference), pp. 143-169. ASTM International (1972)

21. Jin, L., Niu, Z., Jin, B.C., Sun, B., Gu, B.: Comparisons of static bending and fatigue damage between 3D angle-interlock and 3D orthogonal woven composites. J. Reinf. Plast. Compos. 31(14), 935-945 (2012)

22. Karayaka, M., Kurath, P.: Deformation and failure behavior of woven composite laminates. J. Eng. Mater. Technol. 116(2), 222 (1994)

23. Michaels, J.E., Michaels, T.E.: Detection of structural damage from the local temporal coherence of diffuse ultrasonic signals. IEEE Trans. Ultrason. Ferroelectr. Freq. Control 52(10), 1769$1782(2005)$

24. Lobkis, O., Weaver, R.: Coda-wave interferometry in finite solids: recovery of p-to-s conversion rates in an elastodynamic billiard. Phys. Rev. Lett. 90(25), 254302-1(4) (2003)

25. Larose, E., Hall, S.: Monitoring stress related velocity variation in concrete with a $2 \times 10^{-5}$ relative resolution using diffuse ultrasound. J. Acoust. Soc. Am. 125(4), 1853-1856 (2009)

26. Zhang, Y.: Controle de santé des matériaux et des structures par analyse de la coda ultrasonore. PhD Thesis, Université de Maine (2013)

Publisher's Note Springer Nature remains neutral with regard to jurisdictional claims in published maps and institutional affiliations. 